\title{
Survey of Cryptocurrency Volatility Prediction Literature Using Artificial Neural Networks
}

\author{
Sina E. Charandabi \\ LeBow School of Business, Drexel University \\ 3220 Market St, Philadelphia 19104, United States \\ E-mail: se456@drexel.edu \\ Kamyar Kamyar (Corresponding author) \\ Department of Economics, The Ohio State University \\ 1945 N High St, Columbus OH 43210, United States \\ E-mail: Kamyar.5@osu.edu
}

Received: December 7, 2021 Accepted: January 15, 2022 Published: January 20, 2022

doi:10.5296/ber.v12i1.19301ＵRL: https://doi.org/10.5296/ber.v12i1.19301

\begin{abstract}
We start by presenting a short description of the concept of cryptocurrency and the history behind it. Recently-developed literature that attempt to predict volatilities of cryptocurrency valuations through creation of hybrid artificial neural network models are then discussed. For the major part of the paper, we delve into details of multiple hybrid artificial neural networks that were thoroughly implemented to predict cryptocurrency volatilities. Results are reported within the form of a survey. Finally, we compare different methods and discuss their results follow at the end.
\end{abstract}

Keywords: Neural networks, Cryptocurrency valuation, Volatility

\section{Introduction}

The last decade has witnessed an enormous hike in usage and popularity of cryptocurrencies. The initial idea of a virtual currency was given by American computer scientist and cryptographer David Chaum in early 80 's. His initiative first being creation of a secure signature system for credit card authorization, his research led to the idea of virtual wallets and currencies. In late 80's he founded the first virtual currency, ecash, which went bankrupt in a decade. 
The idea of a decentralized cryptocurrency emerged by Nakomoto in the original 2008 Bitcoin paper. Records indicate that the first Bitcoin transactions were performed in early 2009, despite the Nakomoto's character/pseudonym having remained unknown to date. Since 2009, All cryptocurrencies, including Bitcoin, have become more and more popular over time. The primary enormous price hikes of Bitcoin, as a result of a sudden increase in demand with supply not increasing equivalently, took place in 2017. Since then, all cryptocurrencies, especially Bitcoin, have been experiencing sudden hikes repetitively.

Such price hikes make Bitcoin and most other cryptocurrencies extremely volatile financial assets in a holistic time-series view. Indeed, short-term investors are enormously interested in forecasting the timelines of such volatilities in order to maximize investment profits. Economic and Financial theory argues that volatilities are due to real-world shocks and/or supply and demand difference surges. However, recent time-series Bitcoin prices show extreme volatilities that may not be explained by classical financial and economic theory.

In recent time-series econometrics literature, there exist research papers that successfully attempt to model and forecast such unexplained volatility (e.g., Andersen et. al., 2003). More recently, cryptocurrency-specific literature has attempted to use artificial neural network methods to predict such volatility. Due to the novel nature of cryptocurrency data prediction research, the literature is thin and has just recently (after 2017) began growing. Throughout this paper, we explain the various methodologies and results used in artificial neural network models predicting cryptocurrency volatility. In conclusion, we compare results and elaborate on available paths for further research. It should be noted that this survey paper doesn't analyze literature focusing on predicting cryptocurrency prices. For a survey of predicting cryptocurrency prices, refer to Charandabi and Kamyar, 2021A.

\section{Predictability of Volatility}

Realized volatility is the time aggregation (normally over a trading day) of the sum of the squares of logarithmic returns on a highly frequent sample. Andersen et. al. (2003) devises a model to predict financial market volatilities in a specific framework. The model is established on the quadratic variance of the theory of arbitrage-free price processes in time series, and it uses connections among realized volatility and matrix of conditional covariance. The model is further tested on German and Japanese currency to USD exchange rates (on an autoregressive Gaussian vector for logarithmic values) and reportedly performs plausibly. The statistical particular details of the model benchmark involve a parametric mixture of lognormal and normal distribution. The mathematical baseline for Realized Volatility model devised by Andersen et. al. are given in relations (1) through (3), with a N-times sampled aggregation period between $i$ and $i+T$ periods.

$$
\begin{aligned}
& \mathrm{RV}(\mathrm{t})=\sum_{i=1}^{n} R_{i}^{2} \\
& \mathrm{R}_{\mathrm{i}}=\log \left(P_{i} / P_{i-1}\right) \\
& \mathrm{P}_{\mathrm{i}}=P(t+i T / N)
\end{aligned}
$$




\section{Macrothink}

Business and Economic Research ISSN 2162-4860 2022, Vol. 12, No. 1

Figure 1 (Miuri et. al., 2019) depicts the log returns' distribution using relations (1) through (3), performed on 1,804,479 values of Bitcoin price data spanning over 6 years (2012 through 2018). Volatility clustering may be observed from the figure.

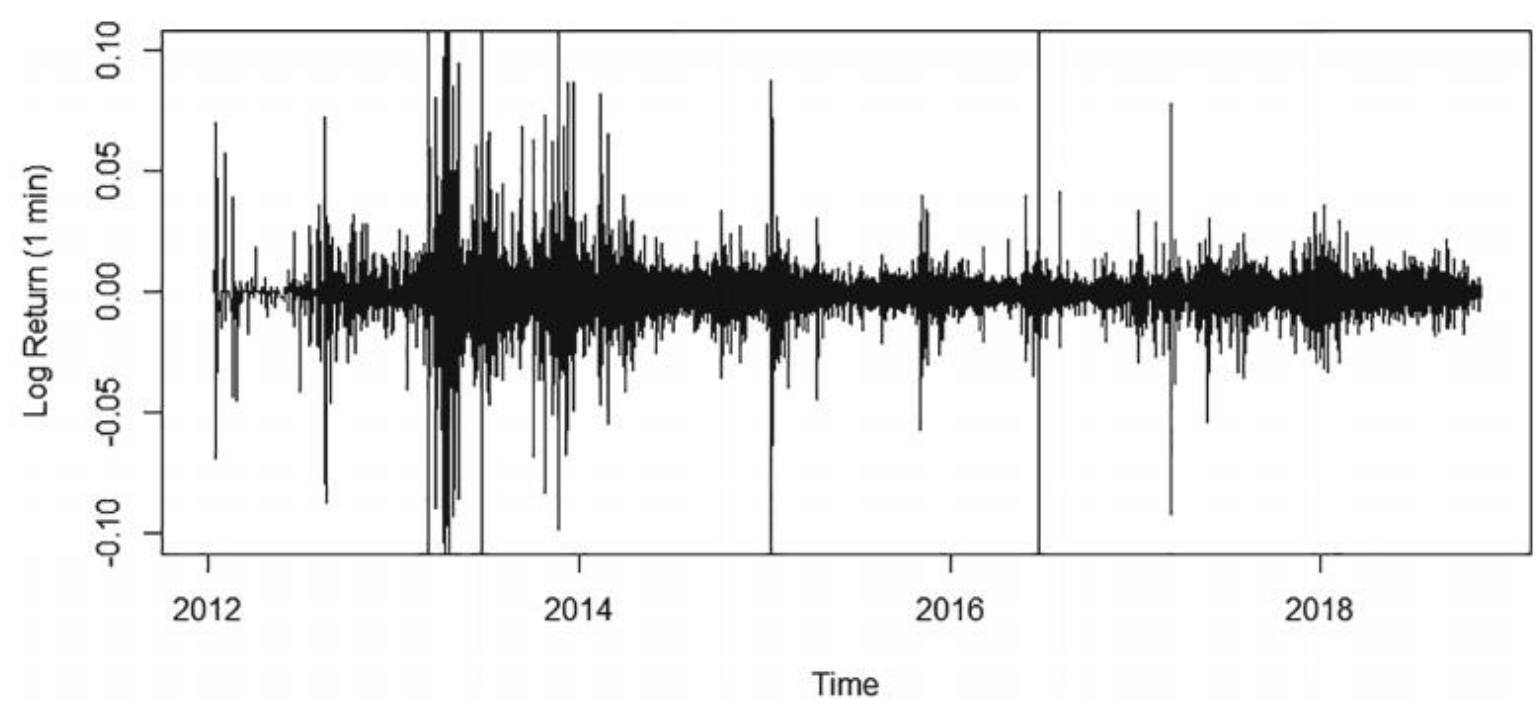

Figure 1. Volatility clustering on logarithmic Bitcoin data on 1,804,479 values spanning over 6 years (Miuri et al., 2019)

\section{Using Long Short Term Memory (LSTM)}

A number of projects have attempted to predict Bitcoin price volatilities in varieties of ANN-LSTM settings using different data sets. Formulas written below depict the theoretical framework for any node. (Zhengyang et al., 2019)

$$
\operatorname{node}(\mathrm{i})=\mathrm{f}\left(\sum_{i=1}^{n} w_{i} x_{i}\right)
$$

in which

$$
\mathrm{f}_{\text {node }}=\frac{1}{1+e^{\text {node }}}
$$

Architectural overview of mechanism of Long Short Term Memory is depicted in figure 2. 

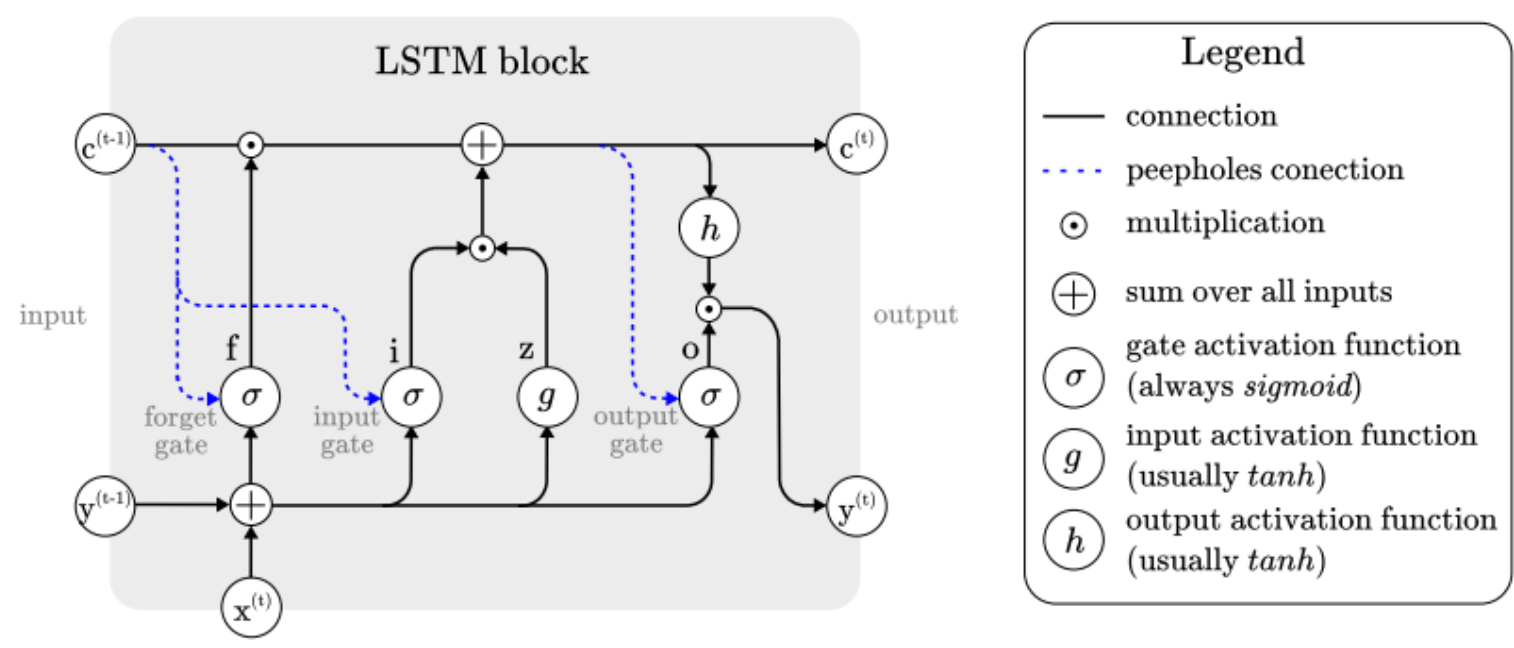

Figure 2. Architectural overview of mechanism of a block of Long Short Term Memory (Van Houdt et al., 2020)

Maiti et al. (2020) attempts to predict volatility in Bitcoin, controlling for co-movement of the volatility in prices of other cryptocurrencies (Monero, XBP, Dash, Stellar, Litecoin, and Etherum). The particular ANN methods used are linear, LSTM, and trained ANN. The proposed trained ANN method has 2 layers $(55 \mathrm{NN}-1 \mathrm{~L}, 16 \mathrm{NN}-2 \mathrm{~L})$ with an activating function of $\operatorname{ReLU}\left(f(x)=\log \left(1+e^{x}\right)\right)$. The particular LSTM model also has two layers (50 LSTM NN- $1 \mathrm{~L}, 16 \mathrm{NN}-2 \mathrm{~L}$ ), with an activating function of second layer - ReLU. Based on the results, the authors find that LSTM yields more plausible results for no lags and/or 0-3, while for large lags $0-7$, the ANN is appropriate. Further, they find that linear models are misleading and inappropriate for the purpose.

Miura et al. (2019) proposes a more detailed approach. Using LSTM blocks with various artificial neural networks algorithms 3-lag (heterogenous AR model of realized volatility (HARRV), perceptron with multiple layers (MLP, dense layer NN), convolutional NN (CNN), long short-term memory (LSTM), gated recurrent unit (GRU), support vector machine (SVM) and ridge regression), Miura et al. (2019) predicts volatilities for bitcoin prices and cross-validates the results in 100 runs. Detailed results are depicted in Table 1. The table includes values for both mean squared error (MSE) as well as the rooted MSE (RMSE), both for the initial test and the cross validation (CV) test. Based on the results, the authors argue that Ridge Regression is the best prediction method. 


\section{MInstitute Macrothink $^{m}$}

Table 1. Results of multiple ANN methods in volatility prediction in Miuri et al. (2019)

\begin{tabular}{|l|l|l|l|l|l|}
\hline Model & Seq. Length & CV-MSE & CV-RMSE & Test-MSE & Test-RMSE \\
\hline HARRV & $1,6,16$ & 3.161 & 1.778 & 4.844 & 2.201 \\
\hline MLP 4 layers with dropout & 10 & 2.961 & 1.720 & $4.870($ var 1.331) & $2.206($ var 6.820) \\
\hline MLP 4 layers with BN & 10 & 3.015 & 1.736 & $4.821($ var 4.999) & $2.195($ var 2.532) \\
\hline LSTM 2 layers + 1 Dense & 12 & 2.984 & 1.727 & $4.823($ var 2.008) & $2.196($ var 1.029) \\
\hline GRU 1 layer + 2 Dense & 5 & $\mathbf{2 . 9 6 0}$ & $\mathbf{1 . 7 2 0}$ & $4.743($ var 8.814) & $2.177($ var 4.578) \\
\hline CNN 2 layers + 1 Dense & 6 & 3.060 & 1.749 & $4.760($ var 1.290) & $2.181($ var 6.760) \\
\hline SVM & 7 & 3.212 & 1.792 & 4.346 & 6.529 \\
\hline Ridge Regression & 6 & 3.061 & 1.749 & 4.666 & 2.160 \\
\hline
\end{tabular}

\section{Other Artificial Neural Network Frameworks}

Among other literature using ANN methods to predict movements is Pabuçcu et al. (2020). Methods used include Support Vector Machine (SVM), Naïve Bayes (NB), Random Forest (RF), and Logistic Regression (LR). The Support Vector Machine method is a non-stochastic method based on a process of minimizing structural risk through maximization of the margin between samples (negative, positive). The Naïve Bayes method is a simplistic method based on the Bayes conditional probability principle Theorem. Allowing to compare results with other algorithms, The Random Forest method is an ensemble-learning algorithm based on a single classifier's incapability of determining the class of test data. Finally, the Logistic Regression is a popular technique modeling the probability of discrete outcomes. For each of the mentioned algorithms, multiple three-parameter combinations were determined and the most plausible of each was chosen for the final test. Table 2 shows the comparison of these methods, with True Positive, False Positive, ROC, and F statistic. The results are further ranked in terms of efficiency, using LR as the benchmark.

Table 2. Comparison of efficiency of ANN methods used in Pabuçcu et al. (2020)

\begin{tabular}{|l|l|l|l|l|l|}
\hline & TP & FP & ROC & F-Stat. & Rank \\
\hline ANN & 0.948 & 0.557 & 0.931 & 0.941 & 1 \\
\hline SVM & 0.948 & 0.610 & 0.669 & 0.938 & 3 \\
\hline NB & 0.882 & 0.167 & 0.901 & 0.902 & 4 \\
\hline RF & 0.946 & 0.557 & 0.923 & 0.939 & 2 \\
\hline LR & 0.858 & 0.873 & 0.681 & 0.854 & (Benchmark) \\
\hline
\end{tabular}

Also establishing on Bayesian Probabilistic theory and principle, Jang et al. (2017) attempts to explain the volatility by proposing an artificial neural network with a multi-layer perceptron maximizing the value of posterior. They use a Bayesian Neural Networks (BNN) algorithm, taking blockchain data spanning from 2011 through 2017 into account, controlling for simultaneous values of stocks prices and currency exchange rates. Their efficiency results are given in Table 3. Given the resulting MAPE value of $1 \%$, their attempt appears to be successful. 
Table 3. Significant Results of BNN method in Jang et al. (2017).

\begin{tabular}{|c|c|c|c|c|c|}
\hline \multirow{2}{*}{ Response var. } & \multicolumn{2}{c|}{$\begin{array}{c}\text { Log } \\
\text { price }\end{array}$} & \multicolumn{2}{c|}{$\begin{array}{c}\text { Log } \\
\text { volatility }\end{array}$} \\
\hline \multirow{2}{*}{ Num. of Input var. } & $\mathbf{2 6}$ & $\mathbf{1 6}$ & $\mathbf{2 5}$ & $\mathbf{1 6}$ \\
\hline \multirow{2}{*}{$\begin{array}{c}\text { Linear } \\
\text { Regression }\end{array}$} & RMSE & - & 0.0935 & - & 0.4823 \\
\cline { 2 - 6 } & MAPE & - & 0.0712 & - & 0.6263 \\
\hline $\begin{array}{c}\text { Bayesian } \\
\text { NN }\end{array}$ & RMSE & 0.0039 & 0.0069 & 0.2546 & 0.2325 \\
\cline { 2 - 6 } & MAPE & 0.0138 & 0.0180 & 0.5090 & 0.5222 \\
\hline \multirow{2}{*}{$\begin{array}{c}\text { Support vec. } \\
\text { Regression }\end{array}$} & RMSE & 0.3201 & 0.2742 & 0.5487 & 0.5297 \\
\cline { 2 - 6 } & MAPE & 0.0428 & 0.0404 & 0.7232 & 0.8629 \\
\hline
\end{tabular}

\section{Autoregressive Model with Jumps}

The autoregressive model with jumps was first introduced in Andersen et al. (2007). It uses the same realized volatility and logarithmic return as in relations (1) and (2), and accounts for bipower variation measures and corresponding nonparametric tests for jumps. Pichl et al. (2017) applies the Anderson methodology to the case of forecasting Bitcoin volatility. The regression equation is given in relation (6), with $J$ values as jumps defined in Andersen et al. (2007). Table 4 shows the regression results for exchange rate of Bitcoin to USD, using 88 days of volatile price data in 2017 with daily 5-min time series. Based on the results, the authors conclude that their proposed regression model captures the dynamics of daily Realized Volatility as aggregated on the 5-minute grid plausibly.

$$
\sqrt{\mathrm{RV}}_{i+1}=\beta_{0}+\beta_{1} \sqrt{\mathrm{RV}}_{i}+\beta_{2} \sqrt{\mathrm{RV}}_{i-5}+\beta_{3} \sqrt{\mathrm{RV}}_{i-10}+\beta_{4} \sqrt{\mathrm{J}}_{i}+\beta_{4} \sqrt{\mathrm{J}}_{i}+\beta_{5} \sqrt{\mathrm{J}}_{i-5}+\beta_{6} \sqrt{\mathrm{J}}_{i-10}
$$

Table 4. Regression results of autoregressive model with jumps (Pichl et al., 2017)

\begin{tabular}{|c|l|l|l|l|l|}
\hline Coefficient & Estimate & Std. error & t-value & p-value & Significance \\
\hline $\boldsymbol{\beta}_{\mathbf{0}}$ & 0.010307 & 0.001459 & 7.065 & $3.22 \mathrm{E}-12$ & $* * *$ \\
\hline $\boldsymbol{\beta}_{\mathbf{1}}$ & 0.344821 & 0.05796 & 5.949 & $3.86 \mathrm{E}-09$ & $* * *$ \\
\hline $\boldsymbol{\beta}_{\mathbf{2}}$ & 0.517929 & 0.115008 & 4.503 & $7.57 \mathrm{E}-06$ & $* * *$ \\
\hline $\boldsymbol{\beta}_{\mathbf{3}}$ & -0.22684 & 0.111337 & -2.037 & 0.0419 & $*$ \\
\hline $\boldsymbol{\beta}_{\mathbf{4}}$ & -0.12326 & 0.077452 & -1.591 & 0.1119 & \\
\hline $\boldsymbol{\beta}_{\mathbf{5}}$ & -0.86087 & 0.147957 & -5.818 & $8.27 \mathrm{E}-09$ & $* * *$ \\
\hline $\boldsymbol{\beta}_{\mathbf{6}}$ & 0.856319 & 0.160656 & 5.33 & $1.24 \mathrm{E}-07$ & $* * *$ \\
\hline
\end{tabular}

\section{Sentiment Analysis}

Valencia et al. (2019) uses scraped data on major cryptocurrencies (Bitcoin, Ethereum, Litecoin, and Ripple) from twitter and the market spanning a 60-day period. The authors note that the scraped tweets were in English, in the given timeframe, not duplicated, and contained the name or symbol of the respective cryptocurrencies. Using Sentiment Analysis techniques 
of Machine Learning, the textual data were numericized and further stacked into Multi-Layer Perceptrons (a certain type of neural networks containing three nodes). Then, using ANN techniques of MLP (Multi-Layer Perceptrons), RF (Random Forest), and SVM (Support Vector Machine), attempts were made to forecast volatilities. Tables 5 and 6 depict results of Valencia et al. (2019) for Bitcoin and Ethereum.

Table 5. Valencia et al. (2019) results for Bitcoin volatility

\begin{tabular}{|l|l|l|l|l|}
\hline Model & Accuracy (95\% CI) & Precision & Recall & $\boldsymbol{F}_{\mathbf{1}}$ Score \\
\hline MLP Twitter & $0.39( \pm 0.02)$ & 0.38 & 0.39 & 0.38 \\
\hline MLP Market & $0.72( \pm 0.03)$ & 0.74 & 0.72 & 0.71 \\
\hline MLP Twitter and Market & $0.72( \pm 0.06)$ & 0.76 & 0.72 & 0.72 \\
\hline SVM Twitter & $0.50( \pm 0.03)$ & 0.29 & 0.50 & 0.37 \\
\hline SVM Market & $0.55( \pm 0.03)$ & 0.53 & 0.56 & 0.47 \\
\hline SVM Twitter and Market & $0.55( \pm 0.03)$ & 0.31 & 0.56 & 0.40 \\
\hline RF Twitter & $0.44( \pm 0.04)$ & 0.50 & 0.80 & 0.62 \\
\hline RF Market & $0.61( \pm 0.04)$ & 0.67 & 0.25 & 0.36 \\
\hline RF Twitter and Market & $0.44( \pm 0.04)$ & 0.28 & 0.44 & 0.34 \\
\hline Random & $0.50( \pm 0.28)$ & 0.49 & 0.50 & 0.50 \\
\hline Majority & $0.55( \pm 0.0)$ & 0.31 & 0.56 & 0.40 \\
\hline
\end{tabular}

Table 6. Valencia et al. (2019) results for Ethereum volatility

\begin{tabular}{|l|l|l|l|l|}
\hline Model & Accuracy (95\% CI) & Precision & Recall & $\boldsymbol{F}_{\mathbf{1}}$ Score \\
\hline MLP Twitter & $0.39( \pm 0.02)$ & 0.44 & 0.39 & 0.38 \\
\hline MLP Market & $0.44( \pm 0.02)$ & 0.44 & 0.39 & 0.35 \\
\hline MLP Twitter and Market & $0.44( \pm 0.03)$ & 0.56 & 0.44 & 0.39 \\
\hline SVM Twitter & $0.39( \pm 0.03)$ & 0.15 & 0.39 & 0.22 \\
\hline SVM Market & $0.39( \pm 0.03)$ & 0.15 & 0.39 & 0.22 \\
\hline SVM Twitter and Market & $0.39( \pm 0.03)$ & 0.15 & 0.39 & 0.22 \\
\hline RF Twitter & $0.33( \pm 0.03)$ & 0.14 & 0.33 & 0.19 \\
\hline RF Market & $0.28( \pm 0.03)$ & 0.12 & 0.28 & 0.17 \\
\hline RF Twitter and Market & $0.39( \pm 0.03)$ & 0.15 & 0.39 & 0.22 \\
\hline Random & $0.50( \pm 0.28)$ & 0.54 & 0.50 & 0.49 \\
\hline Majority & $0.61( \pm 0.0)$ & 0.37 & 0.61 & 0.46 \\
\hline
\end{tabular}

\section{Discussion and Conclusion}

As shown in the previous sections, the purpose of this survey paper is to present and compare the existing literature employing multiple artificial neural network-based approaches to predict cryptocurrency price volatilities. Noting the pros and cons of each of the methods presented (in terms of time elapsed, prediction accuracy, RMSE, MAPE, and R-squared), it appears that in a holistic manner the methods used in Jang et al. (2017), and the Ridge Regression method in Miuri et al. (2019) are of significant efficiency.

Due to the novel nature of this literature, every paper written in the area spans solely to the last three years. Therefore, we may expect many additional papers be written in the years to come. There exist some aspects of cryptocurrency price volatility forecasting with no current existent research papers. An area of possible further work, for example, is to focus solely on implementing data from less volatile cryptocurrencies (e.g. Ethereum, XRP, Chainlink, etc.) 
into the same frameworks. Indeed, many other frameworks could be implemented to forecast and/or measure the prediction efficiencies as well. For instance, the methodology in Filabadi and Mahmoudzadeh (2019) can be implemented to yield a novel model.

Because of the eminent recent hike in the popularity and price of cryptocurrencies (including but not limited to Bitcoin), introduction to the background, history, and technicalities of cryptocurrency is of critical importance to policymakers and other economic agents. The first transaction using bitcoins was to buy a pizza pie a decade ago, which would have been worth over $\$ 5$ million today. With such quick development, all agents need to engage as fast as possible (Charandabi and Kamyar, 2021A).

\section{References}

Andersen, T. G., Bollerslev, T., Diebold, F., \& Labys, P. (2003). Modeling and forecasting realized volatility. Econometrica, 71, 579-625. https://doi.org/10.1111/1468-0262.00418

Andersen, T. G., Bollerslev, T., \& Diebold, F. X. (2007). Roughing it up: including jump components in measuring, modeling and forecasting asset return volatility. Rev Econ Stat, 89, 701-720. https://doi.org/10.1162/rest.89.4.701

Asadi, A., Giahi, R., Filabadi, M. D., Ardakani, A. T., \& Azadeh, A. (2022). A New Stochastic Model for Bus Rapid Transit Scheduling with Uncertainty. Future Transportation, 1(2).

MercoPress. (2021). Bitcoin legal tender in El Salvador, first country ever. [Online] Available: https://en.mercopress.com/2021/06/10/bitcoin-legal-tender-in-el-salvador-first-country-ever

Böhme, R., Christin, N., Edelman, B., \& Moore, T.(2015). Bitcoin: Economics, Technology, and Governance. Journal of Economic Perspectives, 29(2), 213-38.

https://doi.org/10.1257/jep.29.2.213

Camenisch, J., Lysyanskaya, A., \& Meyerovich, M. (2007). Endorsed E-Cash. 2007 IEEE Symposium on Security and Privacy (SP '07). pp. 101-115.

https://doi.org/10.1109/SP.2007.15

Cocco, L., Tonelli, R., \& Marchesi, M. (2021). Predictions of bitcoin prices through machine learning based frameworks. PeerJ Computer Science, 7, e413.

https://doi.org/10.7717/peerj-cs.413

Charandabi, S. E., Ghashami, F., \& Kamyar, K. (2021). US-China Tariff War: A Gravity Approach. Business and Economic Research, 11(3). https://doi.org/10.5296/ber.v11i3.18757

Charandabi, S. E., \& Kamyar, K. (2021). Using A Feed Forward Neural Network Algorithm to Predict Prices of Multiple Cryptocurrencies. European Journal of Business and Management Research, 6(5), 15-19. https://doi.org/10.24018/ejbmr.2021.6.5.1056

Charandabi, S. E., \& Kamyar, K. (2021). Prediction of Cryptocurrency Price Index Using Artificial Neural Networks: A Survey of the Literature. European Journal of Business and Management Research, 6(6), 17-20. https://doi.org/10.24018/ejbmr.2021.6.6.1138

Chaum, D. (1983). Blind signatures for untraceable payments. In D. Chaum, R. L. Rivest \& 
A. T. Sherman (Eds.), Advances in Cryptology - Proceedings of CRYPTO '82 (pp. 199-203). Plenum Press. https://doi.org/10.1007/978-1-4757-0602-4_18

Chaum, D., Fiat, A., \& Naor, M. (1990). Untraceable Electronic Cash. In: Goldwasser S. (eds) Advances in Cryptology - CRYPTO' 88. Lecture Notes in Computer Science, vol 403. Springer, New York, NY. https://doi.org/10.1007/0-387-34799-2_25

Dehghani Filabadi, M. (2019). Robust optimization for SCED in AC-HVDC power systems. Master's thesis, University of Waterloo.

Dehghani Filabadi, M., \& Bagheri, P. (2021). Robust-and-Cheap Framework for Network Resilience: A Novel Mixed-Integer Formulation and Solution Method. arXiv e-prints, arXiv-2110.

Filabadi, M. D., \& Mahmoudzadeh, H. (2022). Effective Budget of Uncertainty for Classes of Robust Optimization. INFORMS Journal on Optimization (in press).

https://doi.org/10.1049/iet-rpg.2019.1127

Filabadi, M. D. (2022). A New Paradigm in Addressing Data Uncertainty: Discussion and Future Research. arXiv e-prints, arXiv-2201.

Filabadi, M. D., \& Azad, S. P. (2020). Robust optimisation framework for SCED problem in mixed AC-HVDC power systems with wind uncertainty. IET Renewable Power Generation, 14(14), 2563-2572.

Ghashami, F., \& Kamyar, K. (2021). Performance Evaluation of ANFIS and GA-ANFIS for Predicting Stock Market Indices. International Journal of Economics and Finance. https://doi.org/10.5539/ijef.v13n7p1

Ghashami, F., Kamyar, K., \& Riazi, S. A. (2021). Prediction of Stock Market Index Using a Hybrid Technique of Artificial Neural Networks and Particle Swarm Optimization. Applied Economics and Finance, 8, 1. https://doi.org/10.11114/aef.v8i3.5195

Ghorbani, N., \& Korzeniowski, A. (2020) Adaptive Risk Hedging for Call Options under Cox-Ingersoll Ross Interest Rates. Journal of Mathematical Finance, 10, 697-704.

https://doi.org/10.4236/jmf.2020.104040

Jang, H., \& Lee, J. (2018).An Empirical Study on Modeling and Prediction of Bitcoin Prices With Bayesian Neural Networks Based on Blockchain Information. IEEE Access, 6, 5427-5437. https://doi.org/10.1109/ACCESS.2017.2779181

Jaquart, P., Dann, D., \& Weinhardt, C. (2021). Short-term bitcoin market prediction via machine learning. The Journal of Finance and Data Science, 7, 45-66.

https://doi.org/10.1016/j.jfds.2021.03.001

Jay, P., Kalariya, V., Parmar, P., Tanwar, S., Kumar, N., \& Alazab, M. (2020). Stochastic Neural Networks for Cryptocurrency Price Prediction. IEEE Access, 8, 82804-82818. https://doi.org/10.1109/ACCESS.2020.2990659

Kamyar, K. (2020). Effect of Unemployment Length on Employment Expectations. 
Undergraduate Economic Review.

Korzeniowski, A., \& Ghorbani, N. (2021). Put Options with Linear Investment for Hull-White Interest Rates. Journal of Mathematical Finance, 11, 152-162.

https://doi.org/10.4236/jmf.2021.111007

Liu, S., Tao, L., Xie, N., \& Yang, Y. (2015). On the new model system and framework of grey system theory. 2015 IEEE International Conference on Grey Systems and Intelligent Services (GSIS). pp. 1-11.

Maiti, M., Vyklyuk, Y., \& Vukovic, D. (2020). Cryptocurrencies chaotic co-movement forecasting with neural networks. Internet Technology Letters, 3, e157.

https://doi.org/10.1002/itl2.157

Madanizadeh, S. A., \& Setayesh, A. (2020). Macroeconomic Uncertainty and Economic Development. Working Paper.

Mehrara, M., Behradmehr, N., Ahrari, M., \& Mohaghegh, M. (2010). FORECASTING VOLATILITY OF CRUDE OIL PRICE USING THE GMDH NEURAL NETWORK. ENERGY ECONOMICS REVIEW, 7(25), 89-112.

Mohaghegh, M., \& Valipour, A. S. (2020). Income-dependent impacts of financial development and human capital on economic growth. A non-stationary panel analysis. Theoretical and Applied Economics.

Mohaghegh, M., \& Valipour, A. S. (2021). Triggering Economic Growth: Trade Liberalization as the Prominent Factor in Less-developed Countries. Business and Economic Research, 11(2), 252-265. https://doi.org/10.5296/ber.v11i2.18491

Miura, R., Pichl, L., \& Kaizoji, T. (2019). Artificial Neural Networks for Realized Volatility Prediction in Cryptocurrency Time Series. In H. Lu, H. Tang \& Z. Wang (Eds.), Advances in Neural Networks - ISNN 2019. Lecture Notes in Computer Science, Springer, Cham. https://doi.org/10.1007/978-3-030-22796-8_18

Nakamoto, S. (2008). Bitcoin: A Peer-to-Peer Electronic Cash System. [Online] Available: https://bitcoin.org/bitcoin.pdf

Pabuçcu, H., Ongan, S., \& Ongan, A. (2020). Forecasting the movements of Bitcoin prices: an application of machine learning algorithms. Quantitative Finance and Economics, 4(4), 679-692. https://doi.org/10.3934/QFE.2020031

Pichl, L., \& Kaizoji, T. (2017). Volatility Analysis of Bitcoin Price Time Series. Quantitative Finance and Economics, 1(4), 474-485. https://doi.org/10.3934/QFE.2017.4.474

Shi, N. (2016). A new proof-of-work mechanism for bitcoin. Financ Innov, 2, 31. https://doi.org/10.1186/s40854-016-0045-6

Sin, E., \& Wang, L. (2017). Bitcoin price prediction using ensembles of neural networks. 13th International Conference on Natural Computation, Fuzzy Systems and Knowledge Discovery (ICNC-FSKD). pp. 666-671. https://doi.org/10.1109/FSKD.2017.8393351 


\section{Macrothink}

Business and Economic Research ISSN 2162-4860 2022, Vol. 12, No. 1

Valencia, F., Gómez-Espinosa, A., \& Valdés-Aguirre, B. (2019). Price Movement Prediction of Cryptocurrencies Using Sentiment Analysis and Machine Learning. Entropy, 21, 589. https://doi.org/10.3390/e21060589

Van Houdt, G., Mosquera, C., \& Nápoles, G. (2020). A review on the long short-term memory model. Artif Intell Rev, 53, 5929-5955. https://doi.org/10.1007/s10462-020-09838-1

Yeganeh, A., Abbasi, S., \& Shongwe, S. (2021). A Novel Simulation-Based Adaptive MEWMA Approach for Monitoring Linear and Logistic Profiles. IEEE Access, 9, 124268-124280. https://doi.org/10.1109/ACCESS.2021.3107482

Yeganeh, A., Shadman, A., Triantafyllou, I., Shongwe, S., \& Abbasi, S. (2021). Run Rules-Based EWMA Charts for Efficient Monitoring of Profile Parameters. IEEE Access, 9, 38503-38521. https://doi.org/10.1109/ACCESS.2021.3061990

Yeganeh, A., Younesi, H. M., Razavian, S., Behzadian, K., \& Shariatmadar, H. (2021). Applying a new systematic fuzzy FMEA technique for risk management in light steel frame systems. Journal of Asian Architecture and Building Engineering.

https://doi.org/10.1080/13467581.2021.1971994

Zhengyang, W., Xingzhou, L., Jinjin, R., \& Jiaqing, K. (2019). Prediction of Cryptocurrency Price Dynamics with Multiple Machine Learning Techniques. In Proceedings of the 2019 4th International Conference on Machine Learning Technologies (ICMLT 2019). Association for Computing Machinery, New York, NY, USA. pp. 15-19.

https://doi.org/10.1145/3340997.3341008

\section{Copyright Disclaimer}

Copyright for this article is retained by the author(s), with first publication rights granted to the journal.

This is an open-access article distributed under the terms and conditions of the Creative Commons Attribution license (http://creativecommons.org/licenses/by/4.0/). 